\title{
Músicas posmodernas: arte sonoro en la Comunitat Valenciana
}

\section{Posmodern musics: sound art in the Valencian Vommunity}

TIPO DE TRABAJO: Comunicación.

\author{
PALABRAS CLAVE
}

Música posmoderna, Arte Sonoro, Comunitat Valenciana.

KEYWORDS

Posmodern music, Sound Art, Comunitat Valenciana.

RESUMEN

Los siglos XX y XXI han sido una de las épocas más revolucionarias de toda la historia del arte. A partir de la era postindustrial, calificada como "tercera revolución industrial", con la creación de nuevos instrumentos electrónicos y la aparición de nuevas tecnologías, las vanguardias musicales de esta época, como el "futurismo", el "experimentalismo", "música electroacústica", etc., comenzarán a considerar en igualdad de condiciones la música, el sonido y el ruido.

A finales de los años setenta, y a partir de dichas propuestas sonoras, algunos artistas utilizarán en sus obras el sonido juntamente con lo visual. Por su parte muchos músicos se adentrarán en lo visual, sobre todo usando artilugios como el video, o similares. Dichas propuestas de unos y otros, recibirán el nombre de "Arte Sonoro".

En esta comunicación intentaremos analizar como los diferentes circuitos tradicionales de música clásica de la Comunidad Valenciana no contemplan en sus programaciones este género musical que supone la confluencia de varias disciplinas artísticas como las instalaciones, performance, paisajes, ambientes, improvisaciones, intervenciones en el espacio sea ciudadano, sea asilvestrado etc. De esta forma los artistas sonoros se han visto obligados a generar un mercado paralelo a los circuitos tradicionales.

\section{ABSTRACT}

The 20th and 21st have been one of the most revolutionary epochs in the history of art. From the post-industrial era, qualified as "third industrial revolution", with the creation of new electronic tools, instruments and the appearance of new technologies, the musical avant-gardes of this era, such as "futurism", "experimentalism", "Electroacoustic music", etc., will begin to consider music, sound and noise in equal conditions.

At the end of the seventies, and based on these sound proposals, some artists will use sound along with visuals in their works. And a lot of experimental musicians will do the same, specially using the video machines as part as their sound instruments. These proposals will receive the name of "Sound Art".

In this communication we will try to analyze how the different traditional circuits of classical music of the Valencian Community do not contemplate in their programming this musical genre that supposes the confluence of several artistic disciplines such as installations, performance, ambient landscapes, etc. In this way sound artists have been forced to generate a parallel market to these traditional circuits.

\section{INTRODUCCIÓN}

La música, a priori, es de todo menos visible. Teniendo en cuenta que el siglo XX, a pesar de ser un siglo devastador por lo que respecta a la falta de humanidad y a la destrucción del ser humano sin un razonamiento justificado, fue también una gran época por lo 
que respecta a la ciencia, el pensamiento y el arte (Watson, 2014). Por lo que respecta a las artes sufren una gran transformación dando lugar al surgimiento de un buen número de tendencias y técnicas diferentes que van a originar una verdadera revolución, no siempre bien aceptadas por todos los públicos y tendencias políticas. La situación política española supuso un estancamiento en los procesos culturales y artísticos. A pesar de esto, algunos "modos de hacer" ${ }^{1}$, más extendidos internacionalmente, y que conocemos actualmente con el nombre global de ARTE SONORO ahondan sus raíces en algunas prácticas anteriores a la época denominada tercera revolución industrial. Dichos "modos de hacer" devienen en una nueva cultura de la imagen y del sonido en la que la característica principal cobra sentido en la convergencia de la interdisciplinariedad de las diferentes artes.

Según Molina, una de las primeras apariciones del término "Arte Sonoro" se atribuye en 1983 en la muestra que tuvo lugar en The Sculpture Center en New York promovida por The Sound Art Foundation, aunque podemos encontrar diferentes artistas que utilizan la música o el elemento sonoro de forma interdisciplinar mucho antes del surgimiento dicha denominación. (2008. p. 214)

Tras estudiar la difusión musical en los últimos años, apreciamos como los circuitos tradicionales de música clásica en la Comunitat Valenciana contemplan unos porcentajes bajísimos en la difusión de las llamadas "músicas contemporáneas", denominación circunscrita exclusivamente a las músicas tardo/orquestales y de cámara, dejando de lado otras praxis que no participan del legado orquestal y académico. Esto supone la creación de unos circuitos paralelos en diferentes instalaciones con el fin de darles una mayor visibilidad. En el caso del Arte Sonoro, ya no se trata solamente de que las instituciones no las contemplen, sino que requieren de otros contextos o parcelas que exceden la clásica sala de conciertos al presentar un pluralismo y eclecticismo propios del posmodernismo. Dicho posmodernismo se refleja musicalmente en el intercambio de los elementos que constituyen las obras sonoras, ya sean elementos plásticos, teatrales, audiovisuales junto con los sonoros y que le dan una pluralidad a sus valores estéticos y a las formas de representación y que se pueden apreciar en las obras que comentamos de los diferentes autores citados en el desarrollo de la investigación del Arte Sonoro en la Comunitat Valenciana. (Paz: 2004. P. 58).

La finalidad de esta investigación es demostrar cómo estos modos de hacer han sido denostados tanto por los circuitos de música como por las instituciones, generando una serie de circuitos paralelos, ricos en contenidos y propuestas, pero pobres en medios, con el fin de dar visibilidad a una serie de artistas que se salen de las prácticas estrictamente académicas.

\section{METOdOLOGÍA}

Hemos realizado un exhaustivo estudio de las fuentes primarias basadas tanto en la música experimental como del arte sonoro tanto en España como en la Comunitat Valenciana.

Además hemos participado en el seminario El Parnàs Emergent organizado por la Banda Primitiva de Llíria y dirigido por Llorenç Barber, mantenido conversaciones con las figuras más importantes involucradas en el objeto de la investigación como el mismo Barber, Montserrat Palacios y Miguel Molina.

\section{DESARROLLO}

Muchos autores afirman que los centros musicales de los años 50 a los 70 del siglo XX eran Madrid y Barcelona, aunque también encontramos creadores muy importantes de ARTE SONORO en la Comunitat Valenciana.

\section{Raíces del Arte sonoro en la Comunitat Valenciana.}

Antes de la aparición del término como tal encontramos creadores que coinciden en la interdisciplinariedad como característica principal, en la que el componente temporal de la música trasciende al ámbito espacial, con un resultado estético completamente innovador de mano de artistas injustamente tratados en el devenir de la historia.

Una figura importante en la evolución de la música experimental será el compositor Eduardo Panach Ramos (1903-1949), quien a principios del siglo XX, investigará y dará a luz una música basada en lo que hoy conocemos como microtonalismo, desglosando así una octava en más intervalos de los doce que suponen el sistema temperado.

\footnotetext{
${ }^{1}$ Terminología utilizada por Llorenç Barber y Montserrat Palacios para referirse a las prácticas artísticas que engloban en su interdisciplinariedad el componente sonoro.
} 


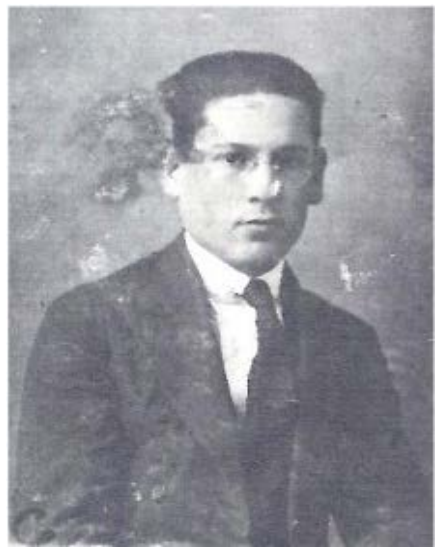

Fig 1: Eduardo Panach Ramos en 1921. Archivo personal del compositor

También encontramos al sacerdote Juan García Castillejo, nacido en 1903 en Motilla del Palancar (Cuenca) y afincado en tierras valencianas, inventor de un aparato bautizado con el nombre de "aparato electrocompositor musical" que generaba de forma automática un tipo de música que surge de la vitalidad de la electricidad y las permutaciones, y que resulta visto desde el presente como un importante antecesor de músicas más actuales.

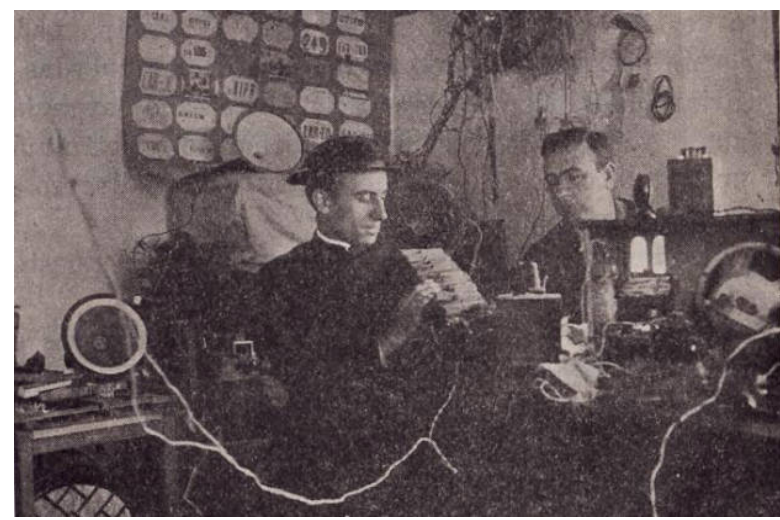

Fig. 2: Juan García Castillejo. Fuente: https://valenciaplaza.com/musica-electronica-pionero-cura-juan-garcia-castillejo

Digno de mención es el granadino Val del Omar, artista polifacético, fundador de Radio Mediterráneo en 1940 y organizador del Primer Circuito Perifónico de Valencia. Patentó en 1944 el sistema de sonido Diafonía y su aparato reproductor Diáfono. ${ }^{2}$

\section{Puentes al presente.}

La situación musical en España tras la guerra civil involucionará hacia estéticas más propias de finales del siglo XIX y principios del XX. Con la llamada Generación del 51, comenzarán a darse a conocer las nuevas corrientes que se estaban gestando fuera del país, aunque posteriormente muchos de ellos moderaran sus estéticas a propuestas más tonales.

El 30 de marzo de 1954 aparece documentada una charla-coloquio organizada por el Instituto Francés de Valencia e impartida por Jean Etienne Marie, titulada "Tendencias más modernas de la música contemporánea", calificada por la crítica como "extravagancia y locura".

\footnotetext{
2 “La diafonía es una estereofonía psíquica donde el espectador está situado entre dos focos, uno delante y otro a su espalda. El de delante expresa el futuro y el de atrás el pasado. Estos dos focos están en choque y se encuentran en el espectador, constituyendo el presente. El eje diafónico enlaza espectador con espectáculo, mientras que el estereofónico está diametralmente opuesto y pasa por los tímpanos de nuestros dos oídos". Declaraciones de Val Omar en Molina, M (2006). Ecos del Arte Sonoro en la vanguardia histórica española en Catálogo de I Muestra de arte sonoro español MASE. Ed. Sensxperiment, Lucena-Córdoba.
} 
El grupo ZAJ, fundado en 1964 por Juan Hidalgo y Ramón Barce junto a Walter Marchetti, dará a conocer el ideario filosófico sobre el sonido de John Cage, contemplando el azar y la aleatoriedad así como las músicas de acción. Posteriormente se irán añadiendo otros artistas de ésta y otras disciplinas como Tomás Marco, Martín Chirino, José Luis Castillejo y Esther Ferrer desarrollando así el mail-art, happenings, la performance y la poesía visual.

Paralelo a ZAJ, en 1966, surgirá el Estudio Alea con el fin de difundir la música contemporánea y cuyos autores y promotores (Luis de Pablo y la familia Huarte) serán los encargados de organizar los Encuentros de Pamplona de 1972. Este pseudo-aperturismo a la situación cultural de la época pondrá de manifiesto la existencia de un grupo de artistas abiertos a las nuevas vanguardias contando con la presencia de artistas internacionales, favoreciendo la multiculturalidad con un sinfín de propuestas en las que se relacionan lo plástico y lo sonoro.

Afincado en Barcelona encontramos al valenciano Carles Santos, quien junto a Mestres Quadreny y la creación del Grup Instrumental Català conformarán sus propios modos de hacer, alejados de los planteamientos de Madrid. De formación clásica, su carrera evolucionará abarcando propuestas como la escultura, la performance, la fotografía, el teatro, el cine y la poesía.

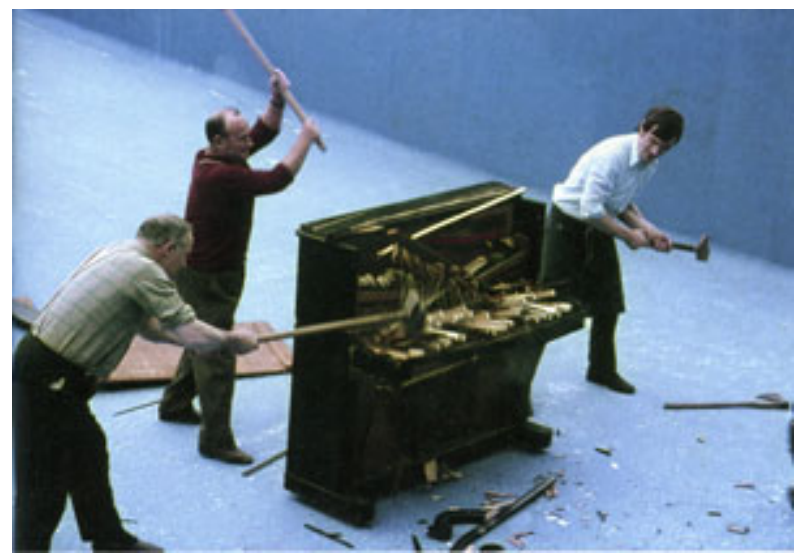

Fig. 3. Fotografía de Clovis Prévost del rodaje para la película Miró sculpteur (1973) de Prévost y Santos. Fuente: Carles Santos Visca el piano! Edición de Manuel Guerrero. P.110

Con la aparición de ACTUM en 1973, de la mano de Llorenç Barber y Josep Lluís Berenguer, comienza a darse a conocer todo tipo de prácticas desconocidas para el público valenciano. ${ }^{3}$ Al igual que $Z A J$, nacerán de la música, abriéndose progresivamente al teatro, John Cage, taller instrumental, pedagogía y sobre todo la electrónica.

ACTUM se dedicará a la difusión de sus propias obras, participando en la creación de ENSEMS en 1979, importante altavoz histórico de difusión de los diferentes modos de hacer culturales y musicales de finales del siglo XX. En dicho encuentro tendrán cabida propuestas que recorren desde lenguajes musicales como el minimalismo, el accionismo, Fluxus, la improvisación, la música concreta y electroacústica, el puntillismo y el estructuralismo, así como la música de acción y body-art, suponiendo una apertura a nuevos lenguajes ampliamente extendidos internacionalmente sin apenas reconocimiento institucional y académico.

Discípulo de Juan Hidalgo, encontramos a Javier Darias, más propio del academicismo musical. Posee un corto periodo creativo situado hasta 1980 aproximadamente, en el que influido por Cage e Hidalgo desarrolla una serie de propuestas poéticas, conceptuales, plásticas y performativas como su Concierto en silencio para piano clausurado, su Sinfonía Supremática o su Envolvente para piano. Esta faceta, transmitida a sus discípulos, queda reflejada en su obra Una Carta a David Tudor.

\footnotetext{
${ }^{3}$ Barber afirma que también estaban en Actum los hermanos Baró, De Nueda, Salvador Porter o Liberto Benet.
} 


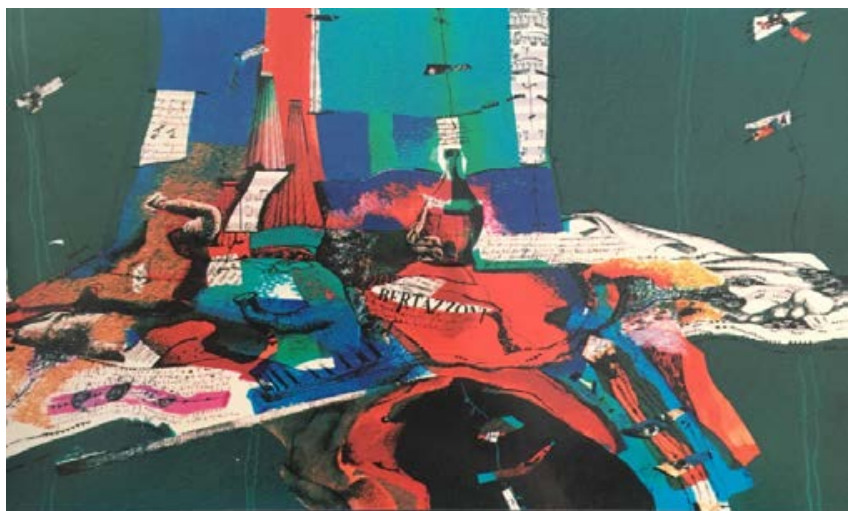

Fig. 4: El laberinto de Lorna, Javier Darias . Oleo collage de Rosana Ara Fuente: Elaboración propia de la portada al libro Cartas a David Tudor de Javier Darias.

\section{Presente y futuro del Arte Sonoro en la Comunitat Valenciana}

Una de las figuras más importantes y reivindicativas del Arte Sonoro pasado, presente y futuro es el ya mencionado Llorenç Barber, participando de forma muy activa desde la creación del grupo ACTUM, la creación de ENSEMS o el festival NITS D'AIELO I ART. Conocido principalmente por sus conciertos de ciudades, músicas volantes, naumaquias, tauromaquias, conciertos para bandas móviles, óperas de papel, escritos y reflexiones, defendiendo unos modos de hacer en los que "la vida y el cosmos son nuestra orquesta", y que no siempre han sido bien entendidos por el academicismo más recalcitrante.

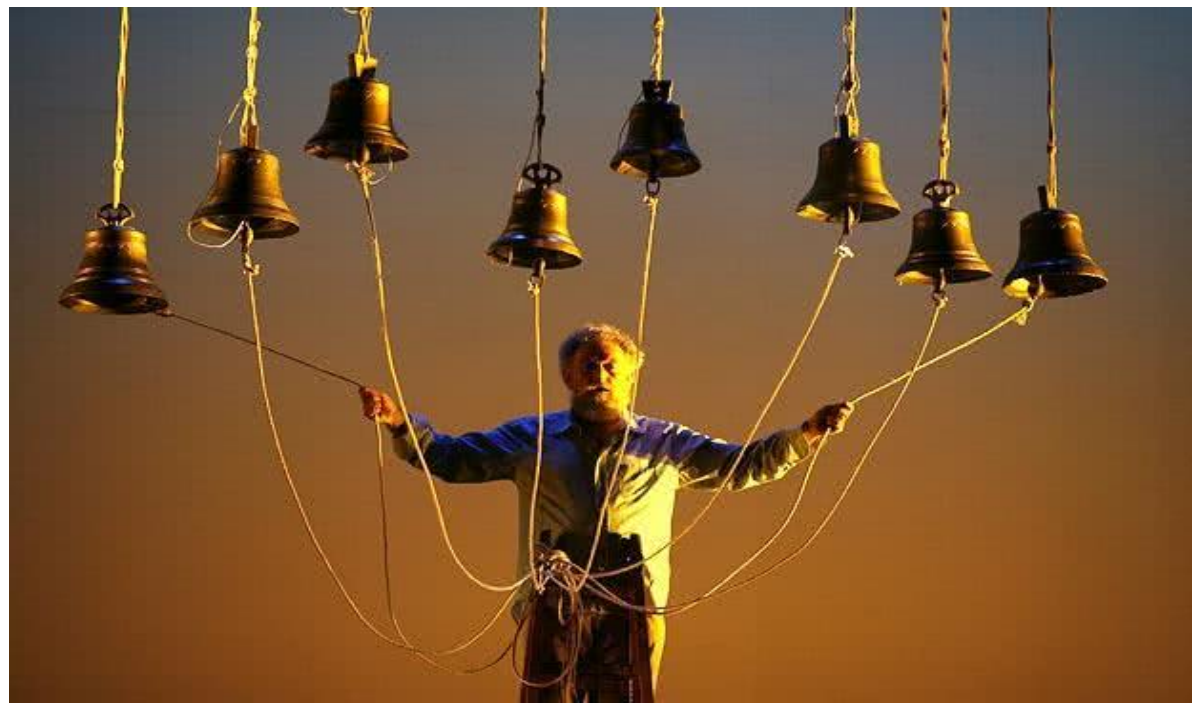

Fig. 5. Llorenç Barber. Fuente: https://www.hoyesarte.com/entrevistas/c32-artistas/llorenc-barber-la-musica-ya-no-se-oye-vestido-de-pinguino-en-la-fila13_121424/

Otro de los artistas valencianos más internacionales es el artista sonoro y compositor José Antonio Orts (Meliana 1955). Alumno de profesores como Blanquer, Berio, Xenakis y Taïra. Destaca en el ámbito del arte electrónico, del arte sonoro y las instalaciones sonoras, estableciendo una perfecta simbiosis entre la escultura y la música. 


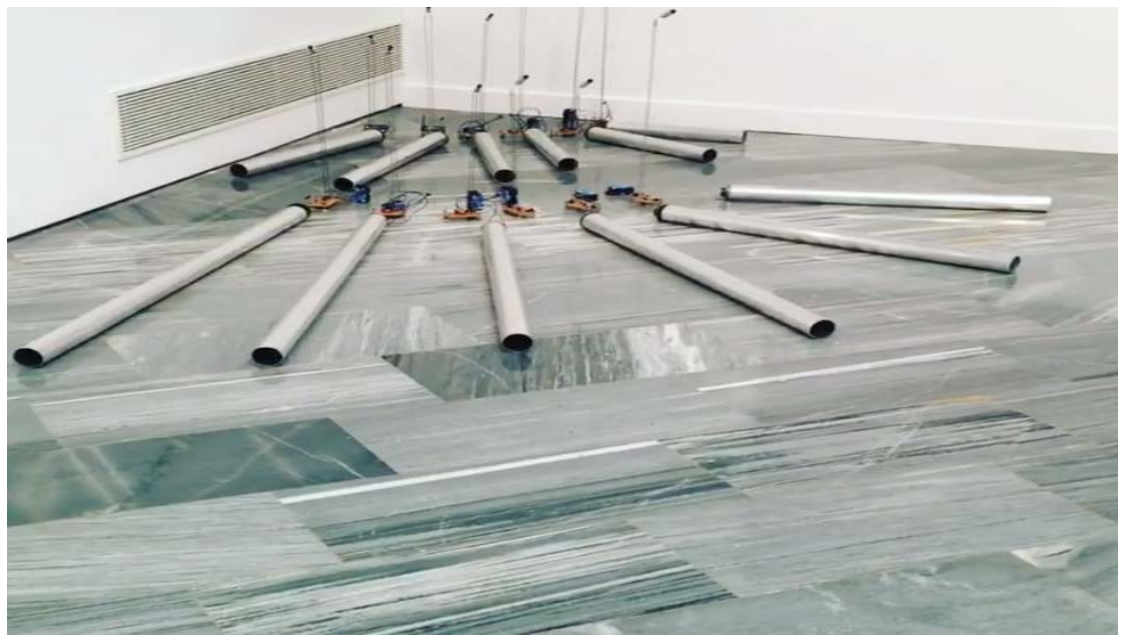

Fig 6: José Antonio Orts: Doble Sexteto. Fuente: Elaboración propia de la Exposición itinerante IVAM CADA Alcoy (2018)

De origen Mexicano pero afincada en Valencia encontramos a la etnomusicóloga, semióloga musical y cantante, especializada en extended voice, Montserrat Palacios. Colaboradora junto a Barber en la redacción del libro: La mosca tras la oreja. De la música experimental al arte sonoro en España (2009). Coautora de la obra radiofónica De campanas y badajos o sobre el amor, encargo de la Saarländischer Rundfunk y compositora de la obra escénico-vocal Coser y cantar.

Miguel Molina Alarcón (Teatinos, 1955) estudia cine, vídeo, música electroacústica y artes plásticas. Es investigador en Arte Sonoro y profesor de la Facultad de Bellas Artes de Valencia, además de uno de los artistas más completos como performer e interventor en espacios públicos. Según Barber: "Molina corre muchos riesgos y tiene una gran habilidad en generar sorpresas". Destaca su instalación sonora Political Show en la exposición Speculation Times (Valencia, 1996). Es también creador del Laboratorio de Creaciones Intermedia (LCI). Sin duda el único engarce del arte sonoro valenciano a una institución de prestigio artístico.

Bartolomé Ferrando (Valencia, 1951), profesor de performance y arte intermedia en la Facultad de Bellas Artes de Valencia además de performer y poeta visual. Cuenta con varias obras sonoras entre las que destaca Flatus Vocis Trio, en la que participa junto a Fátima Miranda y Barber. Flatus Vocis, considerado el mejor grupo de Poesía Sonora a nivel nacional, nace de la unión del texto poético juntamente con los modos de hacer de ACTUM.

Destacan también Ferrer-Molina, David Alarcón, Josep Lluís Galiana y Óscar Vidal.

\section{Lugares de acción}

Dichas propuestas sonoras, mayormente auto-gestionadas, han generando algunos de los festivales más importantes del país como ENSEMS, el cual, a partir del traslado de Barber a Madrid, cobrará un cariz más académico. Como señala Orts: "La evolución del Festival en estos veinticinco años es representativa de la evolución de la música contemporánea en estos mismos años, pero también de la evolución personal de aquellos que han tenido una determinada relación con este" (Ruvira: 2004. P. 65). Carles Santos pondrá en duda la influencia del festival tanto en la realidad musical de la Comunitat Valenciana como en su público, a pesar de ser el festival internacional más antiguo de toda España.

Barber dará a luz al festival, Anem Anem de Gandía, así como la fundación de la Irregularis Daniel Charles Orchestra, que esparcirá por Valencia toda la improvisación más espontánea y simultaneísta. Así mismo junto a Palacios creará el Centre El Cant del Cantó dónde tendrán lugar talleres, pedagogías y la Festa de la Boca donde tendrán cabida todo tipo de MÚSICAS HABLADAS.

Destacan los festivales Poesía Experimental, Ara, organizado por el grupo Texto Fonético, así como el evento que tuvo en lugar en Valencia en 1987 Tramesa d'Art a Favor de la Creativitat en el que participarían Flatus Vocis Trio, representantes de otros países y el mismo Joan Brossa.

En 1989 tuvo lugar en la ciudad de Peñíscola el I Festival Internacional de Performance i Poesia d'Acció organizado por Bartolomé Ferrando. La Universidad de Valencia junto al grupo de percusión Amores, en el año 1999 organizaría los conciertos Arts Uni, en los que tendría cabida tanto la música como la danza, la pintura y la escultura. 
Destaca también el festival ((VIBRA)) Cicle d'Art Sonor i Música Exerimental (Valencia 2006-2011), o el encuentro Vibrato, Arte Sonoro y Experimental (Elche 2009-2010).

El último foco de difusión de Arte Sonoro viene de la mano de Barber quien juntamente con la Banda Primitiva de Llíria y el IES Lluís Vives han consolidado el seminario El Parnàs Emergent que pretende familiarizar al público en general y a los músicos de las bandas en especial de las distintas virtus de los nuevos materiales, técnicas y modos de hacer. Dicha propuesta supone un acercamiento entre el mundo de las bandas y las nuevas músicas.

\section{CONCLUSIONES}

El Arte Sonoro ha supuesto unos nuevos modos de hacer que trascienden el ámbito temporal donde se aúnan diferentes prácticas artísticas gracias a la gran revolución tecnológica.

Estos, mayormente desatendidos por los circuitos tradicionales y por las mismas instituciones, optan así por la creación de circuitos paralelos autogestionados con el fin de dar visibilidad a sus obras a través de los encuentros y festivales mencionados.

La Comunitat Valenciana, conocida internacionalmente por el mundo de las bandas, también debe serlo por desarrollar grandes figuras clave del Arte Sonoro a nivel internacional, y es por eso que debemos ponerlos en el lugar que se merecen.

\section{FUENTES REFERENCIALES}

Barber, LI., y Palacios, M. (2009). La mosca tras la oreja. De la música experimental al arte sonoro en España. Madrid: Ediciones y Publicaciones autor S.R.L. Colección Exploraciones.

Darias, J. (1992). Una carta a David Tudor. Madrid: Ed. Musicinco, S.A.

Guerrero, M. (Ed.) (2006). Carles Santos, Visca el piano! Barcelona: KRTU, Fundació Joan Miró, Generalitat de Catalunya.

Gil Noe, J.V. (2016). Prácticas musicales y sonoras experimentales en Valencia durante el siglo XX, 1922-1983. De olvidadas a precedentes del arte sonoro valenciano. Tesis Doctoral en: http://roderic.uv.es/handle/10550/53909

Iges, J. (2016). Conferencias sobre Arte Sonoro. Madrid: Árdora Ediciones S.L.

López Rodríguez, J. M. (2018). Del colapso tonal al arte sonoro. Un recorrido por la música contemporánea. Punto de Vista Editores.

López, J. (1988). La música en la posmodernidad. Ensayo de hermenéutica cultural. Barcelona: Editorial Anthropos.

Merino Martínez, A. (2017). Eco en el silencio. Surgimiento y desarrollo de un arte sonoro en España. Madrid: Ed: EN ACCIÓN!

Molina Alarcón, M. (2006). Ecos del Arte Sonoro en la vanguardia histórica española. En Catálogo de I Muestra de arte sonoro español MASE. Lucena-Córdoba: Ed. Sensexperiment.

Molina Alarcón, M. (2008). El Arte Sonoro. Itamar, revista de investigación musical: territorios para el arte, 1, 213-234.

Paz Barahona, C. (2004). Los sonidos de la posmodernidad: Los espacios musicales y la historia del presente. Revista Estudios, 18-19, págs. 58-64.

Ruvira, J. (2004). 1979 ENSEMS 2003. 25 Años de Música Contemporánea. Valencia: IVM. GV. 